\title{
Amputation for Sarcoma: Revisiting a 19th Century Treatment in the 21st Century
}

\author{
Derek J. Erstad, $\mathrm{MD}^{1}$ and Chandrajit P. Raut, MD, $\mathrm{MSc}^{2}$ \\ ${ }^{1}$ Department of Surgery, Massachusetts General Hospital, Harvard Medical School, Boston, MA ; ${ }^{2}$ Department of Surgery, \\ Center for Sarcoma and Bone Oncology, Dana-Farber Cancer Center, Brigham and Women's Hospital, Harvard Medical \\ School, Boston, MA
}

Major amputation was once a mainstay of therapy for extremity sarcoma (ES). Wardrop published the first illustration of amputation for a fungating "soft cancer" in 1809. ${ }^{1}$ A critical step in the transition from amputation to limb-sparing surgery was the recognition that inherent tumor biology, rather than local disease control, was the primary determinant of overall outcome. In 1982, Rosenberg and colleagues published a seminal, randomized controlled trial evaluating limb-sparing surgery with adjuvant radiotherapy compared with major amputation. ${ }^{2}$ There was no difference in disease-specific survival (DSS) or overall survival (OS) between treatment groups. Subsequent improvements in surgical technique, including softtissue flap reconstruction and nerve and vessel grafting, allowed for more aggressive resections while preserving critical structures and, importantly, limb function, expanding the range of tumors operable with limb-sparing techniques. ${ }^{3}$ In addition, advances in neoadjuvant/adjuvant therapies including external-beam radiotherapy (EBRT) and brachytherapy expanded treatment options while providing excellent local disease control, reducing the need for reoperation and recurrent treatment. ${ }^{4,5}$ Finally, isolated limb perfusion with melphalan and tumor necrosis factor alpha (TNF $\alpha$ ), the latter not Food and Drug Administration (FDA) approved in the USA, has also been shown to provide value regarding local control for inoperable or locally advanced ES for patients treated in Europe. ${ }^{6}$

(C) Society of Surgical Oncology 2017

First Received: 3 October 2017;

Published Online: 27 November 2017

C. P. Raut, MD, MSc

e-mail: craut@bwh.harvard.edu
Amputation is rarely indicated for primary management of ES in the modern era. Nonetheless, a subset of patients still undergoes amputation, though the current indications and outcomes are not well delineated. This issue of Annals of Surgical Oncology includes two studies that further clarify the contemporary role of amputation for ES. Smith and colleagues from the Royal Marsden Hospital in London, UK analyzed their experience of all major amputations for ES over a 10-year period to provide an upto-date assessment of amputation indications and outcomes. $^{7}$ Van Houdt and colleagues from Mount Sinai Hospital and University of Toronto in Toronto, Canada focused specifically on the utility of hindquarter amputation for proximal lower extremity sarcoma. ${ }^{8}$

Between 2004 and 2014, 69 patients underwent major amputation at the Royal Marsden Hospital (including 23 for primary localized disease from 556 patients undergoing curative-intent surgery, for an amputation rate of $4.1 \%$ ). Overall, $85.5 \%$ of amputations were performed with curative intent (primary localized $33.3 \%$, or recurrent localized, $52.2 \%$ ) while $14.5 \%$ had metastatic disease. The most common indications for amputation in patients with primary localized disease were extensive involvement of the limb girdle, multiple muscle compartment involvement, multifocality, and unsuitability for limb-sparing surgery, typically due to lack of a salvage option. Indications were similar for recurrent localized disease. Tumor size was significantly larger for patients undergoing primary amputation compared with those with recurrent disease (16.0 cm vs. $9.3 \mathrm{~cm}, p=0.007)$. In the recurrent disease group, $100 \%$ had undergone prior surgery in the tumor bed, $75 \%$ were previously treated with local EBRT, and $25 \%$ had already received isolated limb perfusion, decreasing the potential for further limb salvage. In contrast, for patients with metastatic disease, palliative amputation was 
performed for very different indications: fungating lesions, intractable pain, and pathologic fracture.

Amputation was generally well tolerated, with only $14.5 \%$ experiencing a Clavien-Dindo grade II-IV morbidity, and only one patient died within 30 days of surgery. For patients with primary and locally recurrent disease, the 3-year local-recurrence-free survival (LRFS) rates were 72.9 and $97.0 \%$, distant-metastasis-free survival (DMFS) rates were 33.4 and $57.2 \%$, and OS rates were 30.5 and $62.8 \%$, respectively $(p=\mathrm{NS})$. The 3 -year OS rate for all patients with localized disease (primary and locally recurrent) was $50.3 \%$, whereas no patients undergoing palliative amputation for metastatic disease survived beyond 24 months (median OS 6 months). When comparing the 23 patients treated with curative-intent amputation for primary localized disease with those treated with curative-intent limb-sparing surgery, patients undergoing amputation had larger tumors $(16.0 \mathrm{~cm}$ vs. $9.0 \mathrm{~cm}$, $p=0.003)$ and higher-grade disease $(69.9 \%$ vs. $41.1 \%$, $p=0.009$ ). There was no difference in LRFS, though patients undergoing amputation had significantly worse DMSF and OS.

In our own experience at Brigham and Women's Hospital and Dana-Farber Cancer Institute, we found that among 54 patients undergoing amputation for ES over a 10-year period, indications for amputation were similar to those reported from the Royal Marsden Hospital. ${ }^{9}$ In addition to the categories described by Smith and colleagues, we also reported another category of patients undergoing amputation-those with distal sarcomas involving the hand or foot. Unlike other patients with primary ES requiring amputation, these hand/foot sarcoma patients generally had small tumors and better OS.

The detailed analysis by Smith and colleagues provides several insights into the role of amputation for ES in the modern era. ${ }^{7}$ For patients with potentially curative disease, most amputations are indicated for large and invasive tumors that preclude limb salvage despite contemporary techniques. We recommend multidisciplinary evaluation for all patients under consideration for potential amputation to fully explore all potential treatment options, and a second-opinion surgical consultation may be useful. Patients treated with amputation have worse survival, presumably due to their inherently worse tumor biology, and most recurrences are detected within the first 2 years following amputation. Thus, these patients should undergo close surveillance. For patients with metastatic disease, amputation solely provides symptomatic relief with little prospect of survival beyond 2 years.

Van Houdt and colleagues provide a focused analysis on 78 patients undergoing hindquarter amputation/external hemipelvectomy between 1989 and $2015 .{ }^{8}$ As is the case of other forms of ES, limb- and girdle-sparing operations for
ES in this location have been shown to provide similar oncologic outcomes compared to hindquarter amputation, though with improved function. However, despite advances in surgical techniques and nonsurgical therapies, a subset of patients is still treated with this morbid operation.

In this series, $62(79 \%)$ patients were treated with curative intent; common indications included extensive neurovascular involvement and lack of a reconstructive option. The remaining $16(21 \%)$ patients with metastatic disease underwent amputation, most commonly for intractable pain or pathologic fracture. There was no difference in clinicopathologic factors between patients presenting with localized versus metastatic disease. As expected, and similar to the findings from Royal Marsden, patients presenting with metastatic disease had significantly worse survival. For patients with localized disease, 5-year LRFS, DMFS, and OS rates were 85, 49, and 41\%, respectively. There was no difference in OS between patients with primary or recurrent localized disease. On univariate analysis, the combination of age $>65$ years and tumor diameter $\geq 15 \mathrm{~cm}$ was associated with significantly worse DMFS [hazard ratio (HR) 2.93] and OS (HR 2.42). The complication rate was high, as expected, with nearly $50 \%$ of patients developing local wound complications, and $6 \%$ of patients died in the hospital.

This Toronto study provides several insights into surgical management of pelvic girdle sarcoma. First, for appropriately selected patients, there is potential for reasonable survival outcomes. In their review, $25 \%$ of patients with localized disease at time of surgery were alive 10 years later. Second, elderly patients with large tumors are likely to do worse; therefore, consideration of palliative therapies may be more appropriate for this subset of patients. Third, patient selection remains critical for this cohort, as wound complication and mortality rates are quite high. In particular, patients with cardiovascular disease or comorbidities that affect wound healing should also be considered for nonoperative interventions. Fourth, postoperative rehabilitation can be lengthy, which should be balanced against expected survival. Finally, for patients under 45 years of age, over half retain good functional status, including ambulation with a cane and resumption of nonstrenuous daily activities.

As both groups of authors acknowledge, these studies have several limitations. Both are retrospective in nature, limited by relatively small sample sizes, underpowered, and subject to inherent bias.

Nonetheless, taken together, the descriptive nature of both studies provides useful insights into indications, outcomes, and reasonable expectations when pursuing amputation for ES in the modern era. Thankfully, amputations are not needed for most sarcomas, as they were in the 1800s and 1900s. Amputation should always be 
considered a procedure of last resort in patients with ES, but may be reasonable in carefully selected patients with localized primary or recurrent disease without limb salvage options. In patients with metastatic disease, the potential palliative benefit should be weighed against potential complications, length of rehabilitation, and the expected short postoperative survival.

\section{REFERENCES}

1. Wardrop J. Observations on fungus haematodes or soft cancer. Edinburgh: G Ramsay; 1809.

2. Rosenberg SA, Tepper J, Glatstein E, Costa J, Baker A, Brennan M, DeMoss EV, Seipp C, Sindelar WF, Sugarbaker P, Wesley R. The treatment of soft-tissue sarcomas of the extremities: prospective randomized evaluations of (1) limb-sparing surgery plus radiation therapy compared with amputation and (2) the role of adjuvant chemotherapy. Ann Surg. 1982;196:305-15.

3. Ferrone ML, Raut CP. Modern surgical therapy: limb salvage and the role of amputation for extremity soft-tissue sarcomas. Surg Oncol Clin N Am. 2012;21:201-13.

4. Pisters PW, Harrison LB, Leung DH, Woodruff JM, Casper ES, Brennan MF. Long-term results of a prospective randomized trial of adjuvant brachytherapy in soft tissue sarcoma. J Clin Oncol. 1996;14:859-68.

5. Yang JC, Chang AE, Baker AR, Sindelar WF, Danforth DN, Topalian SL, DeLaney T, Glatstein E, Steinberg SM, Merino MJ, Rosenberg SA. Randomized prospective study of the benefit of adjuvant radiation therapy in the treatment of soft tissue sarcomas of the extremity. J Clin Oncol. 1998;16:197-203.

6. Eggermont AM, Schraffordt Koops H, Klausner JM, Kroon BB, Schlag PM, Lienard D, van Geel AN, Hoekstra HJ, Meller I, Nieweg OE, Kettelhack C, Ben-Ari G, Pector JC, Lejeune FJ. Isolated limb perfusion with tumor necrosis factor and melphalan for limb salvage in 186 patients with locally advanced soft tissue extremity sarcomas. The cumulative multicenter European experience. Ann Surg. 1996;224:756-64; discussion 764-755.

7. Smith HG, Thomas JM, Smith MJF, Hayes AJ, Strauss DC. Major amputations for extremity soft-tissue sarcoma. Ann Surg Oncol. 2017. https://doi.org/10.1245/s10434-017-5895-2.

8. van Houdt WJ, Griffin AM, Wunder JS, Ferguson PC. Oncologic outcome and quality of life after hindquarter amputation for sarcoma: is it worth it? Ann Surg Oncol. 2017. https://doi.org/10. 1245/s10434-017-5806-6.

9. Erstad DJ, Ready J, Abraham J, Ferrone ML, Bertagnolli MM, Baldini EH, Raut CP. Amputation for extremity sarcoma: contemporary indications and outcomes. Ann Surg Oncol. 2017. https://doi.org/10.1245/s10434-017-6240-5. 